\title{
A Study on the Teaching of Advanced Chinese Listening from the Perspective of Relevance Theory
}

\author{
Chunjie Wang ${ }^{1,2, *}$ \\ ${ }^{1}$ School of Humanities, Communication University of China, Beijing 100024, China, \\ ${ }^{2}$ School of Humanities and Communication, Anshan Normal University, Anshan, Liaoning, China \\ *Corresponding author. Email: jie_882003@163.com
}

\begin{abstract}
Based on the communication view and context view of relevance theory, this paper makes a statistical analysis on the number of words and sentences, themes, expression methods, number of program codes, option correlation and the correlation between recording content and option in the first part of HSK-6 past exam papers of listening, and puts forward some suggestions for the teaching of advanced Chinese listening, such as encouraging students to read Chinese materials widely, cultivating students' reasoning ability based on options, carrying out teaching for listening in different expressions, and combining conceptual meaning with procedural meaning.
\end{abstract}

Keywords: Relevance theory, Advanced Chinese listening teaching, HSK-6 past exam paper of listening, Teaching suggestions.

\section{INTRODUCTION}

Relevance theory is a theory of verbal communication proposed by Sperber and Wilson. Listening test is an important way to test language learners' communicative competence. With the use of relevance theory, this paper analyzes Chinese listening test questions, clarifies the characteristics of verbal communication in the test questions, and guides Chinese listening teaching, which is helpful to cultivate and improve Chinese learners' Chinese communication ability. HSK, as an international standard exam to test the Chinese level of nonnative Chinese learners, makes a quantitative analysis of its past listening test questions and puts forward corresponding teaching suggestions, which can guide Chinese listening teaching and Chinese listening learning.

According to Zhang Jintao (2005)[1], relevance theory plays a guiding role in the listening of teaching Chinese as a foreign language. Combined with listening textbooks and listening test

*Fund: This paper is supported by the achievements of the expansion project of the special scientific research topic of "14th five-year plan" in Anshan Normal University (2021) -

"Exploration on the training path of applied talents in international education of Chinese language". questions, he put forward four teaching strategies, such as encouraging students to construct relevance chain, using cognitive context for teaching, selecting relevant materials and guiding students to deduce speech intention. Based on relevance theory, Ren Haibo and Zhu Di (2015) [2] proposed that intermediate Chinese listening comprehension should focus on the three elements of "reasoning", "context" and "relevance", and pay attention to training objectives, such as "understanding in context", "looking for the best relevance" and "understanding the real intention". In Jiang Ying's opinions (2016) [3], it is necessary to pay attention to the learning of Chinese vocabulary and cultural background and cultivate students' reasoning and prediction ability based on HSK-3 past exam papers.

The above researches are qualitative researches on Chinese listening teaching with the use of relevance theory. There is no result of quantitative researches on Chinese listening teaching with the use of relevance theory. Under the guidance of relevance theory, this paper makes a quantitative analysis of the first part of listening in the five sets of HSK-6 past exam papers in 2018 [4], and puts forward relevant suggestions on advanced Chinese listening teaching. 


\section{CORE VIEWPOINTS OF RELEVANCE THEORY}

Sperber and Wilson put forward relevance theory in "Relevance: communication and cognition" in 1986, and revised it in the second edition in 1995. Starting from people's cognitive characteristics and processes, relevance theory regards communication as a cognitive activity and holds that human cognition has a general goal, that is, trying to obtain the maximum cognitive effect with the minimum investment in the cognitive process. In order to achieve this goal, people must focus their attention on the most relevant information (He Ziran, 2006:173) [5].

\subsection{Communicative View}

Sperber and Wilson believe that there are two modes of language communication: code and reasoning. These two modes exist simultaneously in language communication, in which the cognitionreasoning process is the basic, and codingdecoding is attached to the cognition-reasoning process. In the cognition-reasoning process, the speaker's discourse expression is an ostensive process, the listener's speech percetion is a reasoning process, and the whole communication process is an ostensive-inferential process. Sperber and Wilson (1995) also proposed that discourse can encode conceptual meaning and procedural meaning. Conceptual meaning plays a role in both explicit and implicit information, and improves the relevance of explicit communication by increasing the relevance hypothesis of discourse; Procedural meaning restricts or guides the process of understanding explicit information and reasoning implicit meaning, so that the listener makes less effort to obtain the maximum contextual effect. (Wu Bingzhang, Xu Shenghuan, 2011:47) [6]

\subsection{Context View}

Sperber and Wilson believe that context is a psychological construct and a series of hypotheses existing in people's brain, so context is also called cognitive context, and its hypothesis is called cognitive context hypothesis. (Jiang Yan, 2008:4048 [7]) Cognitive context is constructed by communicators in the process of communication, which is dynamic. The existing language, logic and encyclopedia play an important role in the construction of context. In the process of discourse understanding, the listener needs to deal with the information or new assumptions provided by the speakers through a series of contextual assumptions, so as to deduce the meaning of the discourse and understand the speaker's communicative intention. (He Ziran, 2006:179)[5]

\section{ANALYSIS OF PAST EXAM PAPERS OF HSK-6 LISTENING}

HSK is an international standard exam to test the Chinese level of non-native Chinese learners, which can be divided into six levels. HSK-6 listening test consists of three parts, of which the first part contains 15 questions. The examination form is as follow: after listening to a short recording, examinees choose one from the four options that is consistent with the listening content, and the recording will only be played once. The examination process of the exam questions is an ostensive-inferential process. The options of test questions and the recorded content play an ostensive role. The examinees need to form contextual assumptions according to the existing language, logic and encyclopedia, make the reasoning in combination with ostensive information, and finally select the best relevant option.

\subsection{The Statistics of Test Question Words}

The test questions are composed of two parts: recording and options. The number of words of each question in the recording part is between 60 and 89 , which is very different. There is little difference in the average number of words per question in each set, ranging from 77 to $81 .{ }^{1}$ The total number of option words in each question is between 27 and 46, and the number of each option words is between 5 and 14, which is very different. There is little difference in the average total number of option words of each question in each set, ranging from 38 to 39 .

\subsection{Statistics of the Number of Sentences in the Test Questions}

The recording part of the test question is a short paragraph with complete semantics, and each paragraph is composed of different number of sentences. The number of sentences in the question of the recording part is between 1 and 4 , which is very different. There is little difference in the

1. The average number of words in the text is rounded to the nearest whole number. 
average number of sentences per question in each set, ranging from 2.4 to $2.5 .^{2}$

\subsection{Statistics of Themes of Test Questions}

The recording content of test questions has a wide range of themes, which can be classified into five categories: science, culture, life, art and education. There are differences in the number of test questions of different themes. The statistics of the number of test questions of different subjects can be seen in "Table 1 ".

It can be seen from "Table 1" that the number of questions on various themes in each set of exam papers is unevenly distributed, the number of questions on science, culture and life is more, and the number of questions on art and education is less.

\subsection{Statistics of Expression Ways of Test Questions}

The recording part of the exam paper is a short paragraph with complete semantics, and each paragraph has its own way of expression. There are three ways of expression in the recording part of exam papers: expository text, argumentation and narrative. The statistics of the expression ways in the recording part of each set of exam papers are shown in "Table 2 ".

It can be seen from "Table 2" that in each set of exam papers, the maximum number of questions in the expression way is between 8 and 12 , the number of questions of argumentation is between 2 and 5 (except exam paper 3), and the number of questions of narrative is (except exam paper 3) between 1 and 3 . The overall proportion of the test questions in three expression ways shows that the test questions of expression way account for highest proportion $(65.3 \%)$, and the test questions of argumentation account for middle proportion (24. $0 \%$ ), and the test questions of narrative account for the least proportion $(10.7 \%)$.

\subsection{Statistics of Test Question Program Coding}

There are two coding methods of concept and program in the recording part of test questions. Because concept coding is relatively common, this paper only makes the statistics of the program

2. The number of sentences in the text is kept to decimal place. coding. Connectives, deixis and parentheses can make the listener pay less effort in the process of discourse understanding. This paper takes these three kinds of words as program coding for statistics. The number of recording program coding of test question is between 0 and 11, with a great difference among questions. The total number of program codes for each set of exam papers is between 58 and 65 , with the average between 3.9 and 4.3. ${ }^{3}$ There is little difference between each set of exam papers. There are differences in the number of recording program codes in different expressions. The statistics of program codes in the recording in different expressions can be seen in "Table 3".

Table 1. Statistics of the number of questions of themes recorded in the first part of HSK-6 listening

\begin{tabular}{|l|l|l|l|l|l|l|}
\hline $\begin{array}{l}\text { Theme Of } \\
\text { Exam Paper }\end{array}$ & One & two & three & four & five & Total \\
\hline Science & 7 & 3 & 2 & 2 & 1 & 15 \\
\hline Culture & 3 & 4 & 5 & 4 & 3 & 19 \\
\hline Life & 4 & 6 & 7 & 8 & 10 & 35 \\
\hline Art & 0 & 1 & 0 & 1 & 1 & 3 \\
\hline Education & 1 & 1 & 1 & 0 & 0 & 3 \\
\hline
\end{tabular}

Table 2. Statistics of the expression ways in the first recording part of each set of exam papers

\begin{tabular}{|l|l|l|l|l|l|l|}
\hline $\begin{array}{l}\text { Expression } \\
\text { ways of exam } \\
\text { paper }\end{array}$ & One & two & three & four & five & Total \\
\hline Narrative & 1 & 1 & 3 & 2 & 1 & 8 \\
\hline Argumentation & 4 & 2 & 2 & 5 & 5 & 18 \\
\hline Expository text & 10 & 12 & 10 & 8 & 9 & 49 \\
\hline
\end{tabular}

Table 3. Statistics of program codes of various expression ways in HSK-6 listening part I

\begin{tabular}{|l|l|l|l|}
\hline $\begin{array}{l}\text { Expression } \\
\text { ways }\end{array}$ & $\begin{array}{l}\text { Number } \\
\text { of } \\
\text { questions }\end{array}$ & $\begin{array}{l}\text { Number of } \\
\text { program } \\
\text { codes }\end{array}$ & $\begin{array}{l}\text { Number of program } \\
\text { codes per question }\end{array}$ \\
\hline Narrative & 8 & 27 & 3.4 \\
\hline $\begin{array}{l}\text { Argumentati } \\
\text { on }\end{array}$ & 18 & 100 & 5.6 \\
\hline $\begin{array}{l}\text { Expository } \\
\text { text }\end{array}$ & 49 & 180 & 3.7 \\
\hline
\end{tabular}

It can be seen from "Table 3" that the value of the program code of test questions in argumentation way is 5.6 (maximum), the value of the program code of test questions in expository way is 3.7 (middle), and the value of the program code of test questions in the narrative form is 3.4 (minimum).

3. In this paper, the number of program code is reserved as 1 decimal place. 


\subsection{Statistics of Question Option Relevance}

Each question in the exam paper has four options. Most of the four options have relevance. The repetition of the same words in different options is the main embodiment of relevance. The statistics of word repetition times are shown in "Table 4".

It can be seen from "Table 4" that the repetition time of the same word ranks from high to low: four times, two times, three times and one time.

There is a strong correlation between word repetition and the expression ways of recorded content. The statistics of word repetition in various expression ways in the exam papers are shown in "Table 5".

It can be seen from "Table 5" that among the options of expository way, the proportion of four times of repetition is the highest and the proportion of one time of repetition is the lowest; among the options of argumentative way, the proportion of two and one time of repetition is high, and the proportion of four and three times of repetition is relatively low; among the options of narrative way, the proportion of two times of repetition is the highest, the proportion of four times of repetition is in the middle, and the proportion of three and one time of repetition is low.

Table 4. Repetition times of the same word in options of HSK-6 listening part 1

\begin{tabular}{|l|l|l|}
\hline Repetition times & number & Proportion \\
\hline 4 & 34 & $45.3 \%$ \\
\hline 3 & 14 & $18.7 \%$ \\
\hline 2 & 18 & $24.0 \%$ \\
\hline 1 & 9 & $12.0 \%$ \\
\hline
\end{tabular}

When there are multiple recurrences of words in the same subtitle option, the number of occurrences of the most recurring words shall prevail.

b One digit is reserved after the decimal point.

Table 5. Statistics of word repetition of various expressions in HSK-6 listening part 1

\begin{tabular}{|l|l|l|l|l|l|l|}
\hline \multirow{2}{*}{ Repetition times } & \multicolumn{2}{|l|}{ Expository text } & Argumentation & \multicolumn{2}{l|}{ Narrative } \\
\cline { 2 - 7 } & number & Proportion & number & Proportion & number & Proportion \\
\hline 4 & 29 & $59.2 \%$ & 3 & $16.7 \%$ & 2 & $25.0 \%$ \\
\hline 3 & 10 & $20.4 \%$ & 3 & $16.7 \%$ & 1 & $12.5 \%$ \\
\hline 2 & 8 & $16.3 \%$ & 6 & $33.3 \%$ & 4 & $50.0 \%$ \\
\hline 1 & 2 & $4.1 \%$ & 6 & $33.3 \%$ & 1 & $12.5 \%$ \\
\hline
\end{tabular}

a The proportion is the proportion of the number of questions repeated in the same expression way to the total number of questions in the same expression.

\subsection{Statistics of the Relevance Between Test Options and Recording Content}

There are four options for each question in the exam paper, and there are 300 options for 75 questions. There are three ways of correlation between options and recorded content: positive correlation, uncorrelation and negative correlation. Positive correlation means that the options are consistent with the recorded content, uncorrelation means that the options are not related to the recorded content, and negative correlation means that the options are related to the recorded content, but there are contradictions. The positive correlation options are the correct options, and the uncorrelation and negative correlation options are the wrong options. The correlation statistics between options and recording content can be seen in "Table 6".
Table 6. Statistics of correlation between options and recorded content in HSK-6 listening part 1

\begin{tabular}{|l|l|l|}
\hline Mode & $\begin{array}{l}\text { Number of } \\
\text { options }\end{array}$ & Proportion \\
\hline positive correlation & 75 & $25.0 \%$ \\
\hline uncorrelation & 140 & $46.7 \%$ \\
\hline negative correlation & 85 & $28.3 \%$ \\
\hline
\end{tabular}

It can be seen from "Table 6" that the proportion of options being not related to the recorded content is $46.7 \%$, and the proportion of options being negatively related to the recorded content is $28.3 \%$.

There are two ways to associate the positive correlation option and negative correlation option with the recorded content: one is only through the concept coding, and the other is through the concept coding and program coding. The statistics of the two correlation methods of the positive correlation option can be seen in "Table 7" and the statistics of the two correlation methods of the 
negative correlation option can be seen in "Table 8".

Table 7. Statistics of correlation between positive correlation options and recorded content in HSK-6 listening part 1

\begin{tabular}{|l|l|l|}
\hline Mode & number & Proportion \\
\hline Concept coding & 9 & $12.0 \%$ \\
\hline $\begin{array}{l}\text { Concept coding } \\
\text { program coding }\end{array}$ & 66 & $88.0 \%$ \\
\hline
\end{tabular}

It can be seen from "Table 7 that among the positive correlation options, the options that are jointly related to the recorded content by concept coding and program coding are the main ones, supplemented by the options that are only related to the recorded content by concept coding.

Table 8. Statistics of correlation between negative correlation options and recorded content in HSK-6 listening part 1

\begin{tabular}{|l|l|l|}
\hline Mode & number & Proportion \\
\hline Concept coding & 52 & $61.9 \%$ \\
\hline $\begin{array}{l}\text { Concept coding } \\
\text { program coding }\end{array}$ & 32 & $38.1 \%$ \\
\hline
\end{tabular}

It can be seen from "Table 8" that among the negative correlation options, only the options of concept coding are related to the recorded content, which are the main, and supplemented by the options of concept coding and program coding that are related to the recorded content.

\section{SUGGESTIONS ON ADVANCED CHINESE LISTENING TEACHING}

Through the statistical analysis of HSK-6 listening part 1 of five sets of exam papers, it is found that there is little difference in the average number of words and sentences in each set of exam papers. The themes of the exam papers come from a wide range, the distribution of test questions of different themes is uneven, the number of test questions in the fields of life, culture and science is large, and the number of test questions in the fields of education and art is small. The uneven distribution of test questions in expression ways shows that the proportion of test questions in expression way is relatively large, the proportion of test questions in argumentation way is in the middle, and the proportion of test questions in narrative way is relatively small. The number of program codes in the test questions is unevenly distributed in the test questions with different expression modes. The number of program codes in the argumentation way is the largest, and the program codes in the expository way and narrative way are relatively small. The frequency of word repetition is not balanced, the proportion of four times of repetition is the highest, the proportion of two times and three times of repetition is in the middle, and the proportion of one time of repetition is the lowest. The frequency of word repetition in the test questions is unevenly distributed in the test questions with different expressions. The proportion of uncorrelation between test options and recorded content is large, the proportion of negative correlation between test options and recorded content is in the middle, and the proportion of positive correlation between test options and recorded content is the smallest. There are differences in the positive correlation and negative correlation between the test options and the recorded content. Based on the above analysis, it is believed that the future advanced Chinese listening teaching should pay attention to the following aspects.

\subsection{Encouraging Students to Read Chinese Materials Widely}

Topics in the field of science in the exam papers include graupel, Beidou system, swordfish, dark matter, Peristeria Elata, etc; topics in the field of life include purifiers, coins, seasonal fruits and vegetables, food preservation, etc; topics in the cultural field include tea, porcelain, cliff painting, lions on raised platform, antler beams, etc. The topics in the exam papers are widely distributed. In normal teaching, students should be encouraged to widely read Chinese materials, especially language materials in the fields of life, science and culture, so as to enrich language, logic and encyclopedia knowledge.

\subsection{Cultivating Students' Reasoning Ability Based on Options}

Options are the first information obtained by students in the process of answering questions, and also are the basis for students to construct cognitive context and form context hypothesis. In ordinary classroom teaching, it is required to guide students to construct cognitive context when reading options. By reading the four options, the topic of the test question can be inferred according to the words of high repetition times. If there are no words of high repetition times, it is necessary to use the existing knowledge to explore the internal relationship of the options and infer the relevant 
contents of the test question. After reading the options, students listen to the questions and verify the constructed contextual hypothesis, and finally obtain the positively related options.

\subsection{Carrying out Listening Teaching for Different Expression Ways}

The expression ways of exam papers are mainly expository text, argumentation and narrative. There are differences in the test questions of different expression methods. The expository text will focus on the description object, the argumentation will focus on the demonstration of the core argument, and the narrative method will focus on the five elements of the narrative. The option setting of test questions with different expressions also has different characteristics, which shows that the repetition rate of test questions in expository way is high, and the nouns with high repetition are generally topics. The repetition rate of verbs in argumentation way is high, and the verbs with high repetition are generally "to..." or "not to...". The repetition rate of names and appellations in narration is high. Teachers carry out special listening teaching for different expressions, which can make students familiar with the writing characteristics and the characteristics of option setting.

\subsection{Carrying out the Teaching in the Combination of Conceptual Meaning and Procedural Meaning}

The recording part of exam papers mostly adopts the combination of concept coding and program coding. Only a few recording parts of exam papers only have concept coding without program coding. The correlation between the test question options and the recorded content is also mostly constructed in the combination of concept coding and program coding. In the teaching process, teachers guide students to combine the conceptual meaning presented by concept coding with the procedural meaning presented by program coding, which can improve students' ability to construct cognitive context and verify contextual assumptions.

\section{CONCLUSION}

Combined with relevance theory, this paper makes a quantitative analysis of the first part of five sets of HSK-6 listening exam papers, and put forward four targeted teaching suggestions on the basis of quantitative analysis, hoping to help advanced Chinese learners improve their Chinese listening ability.

\section{AUTHORS' CONTRIBUTIONS}

This paper is independently completed by Chunjie Wang.

\section{REFERENCES}

[1] Zhang Jintao. "The Application of Relevance Theory in Teaching Chinese as a Foreign Language" [D], Jilin University, 2005. (in Chinese)

[2] Ren Haibo, Zhu Di. "The Basics, Elements and Goals of Intermediate Chinese Listening Comprehension: Understanding Based on Relevance Theory" [C] "International Chinese Language Education Research", 2015, 28-34. (in Chinese)

[3] Jiang Ying. "Teaching Strategies for Teaching Chinese as a Foreign Language from the Perspective of Relevance Theory: Taking HSK Intermediate Exam Papers as an Example" [J], "Vocational Education", 2016, Issue 26. (in Chinese)

[4] Confucius Institute Headquarters/Hanban. "Collection of Chinese Proficiency Test (HSK-6)" [Z], Beijing: People's Education Press, (2018. (in Chinese)

[5] He Ziran. "Cognitive Pragmatics-A Cognitive Study of Verbal Communication" [M] Shanghai: Shanghai Foreign Language Education Press, 2006. (in Chinese)

[6] Wu Bingzhang, Xu Shenghuan. "Cognitive Pragmatics Research" [C] Shanghai: Shanghai Foreign Language Education Press, 2001. (in Chinese)

[7] [France] Dan Sperbo, [U.K.] Die Jue Wilson. "Relevance: Communication and Cognition" [M], Jiang Yan Trans. Beijing: China Social Sciences Press, 2008. (in Chinese) 